Article

\title{
Upper Bound Design for the Lipschitz Constant of the $F_{\mathrm{G}}(v, \mathrm{q})$-Entropy Operator
}

\author{
Yuri S. Popkov 1,2
}

1 Institute for Systems Analysis of Federal Research Center "Computer Science and Control" of Russian Academy of Sciences, Moscow 119333, Russia; popkov@isa.ru

2 Department of Software Engineering, Braude College of Haifa University, Karmiel 2161002, Israel

Received: 12 March 2018; Accepted: 17 April 2018; Published: 7 May 2018

\begin{abstract}
This paper develops an upper bound design method of the Lipschitz constant for the generalized Fermi-Dirac information entropy operator with a polyhedral admissible set. We introduce the concept of a normal operator from this class in which the constraint matrix has normalized columns. Next, we establish a connection between the normal and original operator. Finally, we demonstrate that the normal operator is majorized by the linear one and find numerical characteristics of this majorant.
\end{abstract}

Keywords: entropy; majorant; normal operator; monotonic operator; vector interval

\section{Introduction}

Mathematical modeling methods based on constrained optimization of parameterized entropy functions (or functionals) are widely used in different applications such as image recognition in computerized tomography [1,2], dynamic regression models estimation [3], and randomized machine learning [4], to name a few.

Entropy functions and admissible sets depend on basic variables and parameters, some being fixed while others take values from their definitional domains. An entropy operator is an operator that maps the definitional domains of variable parameters into a set of entropy-optimal basic variables. In general form an entropy operator can be written as

$$
\begin{gathered}
\mathbf{u}(\mathbf{v}, \mathbf{g})=\arg \max _{\mathbf{u}}(H(\mathbf{u}, \mathbf{v}) \mid \mathbf{u} \in \mathcal{D}(\mathbf{g})), \\
\mathbf{u} \subset R_{+}^{m}, \mathbf{v} \in \mathcal{V} \subset R_{+}^{m}, \mathbf{g} \in \mathcal{Q} \subset R_{+}^{r},
\end{gathered}
$$

where $H(\mathbf{u}, \mathbf{v})$ denotes an entropy function of basic variables $\mathbf{u}$ and parameters $\mathbf{v}$ while $\mathcal{D}$ gives an admissible set with parameters $\mathbf{g}$.

This paper deals with the so-called $F_{\mathbf{G}}(v, \mathbf{q})$-entropy operators defined by

$$
\begin{gathered}
\mathbf{u}^{F}(v, \mathbf{q})=F_{\mathbf{G}}(\nu, \mathbf{q})=\arg \max _{\mathbf{u}}\left(H_{F}(\mathbf{u}, v) \mid \mathbf{u} \in \mathcal{D}(\mathbf{q})\right), \\
\mathbf{u} \subset R_{+}^{m}, v \in \mathcal{N} \subset R_{+}^{m}, \mathbf{q} \in \mathcal{Q} \subset R_{+}^{r},
\end{gathered}
$$

where $H_{F}(\mathbf{u}, v)$ denotes the generalized Fermi-Dirac information entropy, $\mathcal{N}$ and $\mathcal{Q}$ are $m$-dimensional unit and $r$-dimensional parallelepipeds, respectively, and

$$
\mathcal{D}(\mathbf{q})=\{\mathbf{u}: B \mathbf{u}=\mathbf{q}\},
$$

where

$$
B \geq 0 .
$$


In what follows, we will suggest an upper bound design method for the local Lipschitz constant (over a compact set) of the entropy operators belonging to this class.

The Lipschitz constant plays an important role in theory of dynamic systems, including for the systems with entropy operator [5], in dynamic procedures of computerized tomography [6], and others.

\section{Problem Statement and Logical Scheme of Solution}

Consider the entropy operator $F_{\mathbf{G}}(v, \mathbf{q})(2)$ with the entropy function

$$
H_{F}(\mathbf{u}, v \mid \mathbf{G})=-\sum_{i=1}^{m} u_{i} \ln \frac{u_{i}}{v_{i}}+\left(G_{i}-u_{i}\right) \ln \left(G_{i}-u_{i}\right),
$$

and the matrix $B$ (3) of full rank $r$. The definitional domains of the variables have the form

$$
\begin{aligned}
\mathcal{S} & =\mathcal{N} \otimes \mathcal{Q}, \\
\mathcal{N} & =\left\{v: 0<\sigma_{i} \leq v_{i} \leq v_{i}^{*}<1, \mid i=\overline{1, m}\right\}, \\
\mathcal{Q} & =\left\{q: 0<\epsilon_{k} \leq q_{k} \leq q_{k}^{*}<\infty, \mid k \overline{1, r}\right\},
\end{aligned}
$$

with constants $\sigma_{1}, \ldots, \sigma_{m}, v_{1}^{*}, \ldots, v_{m}^{*}$, and $\epsilon_{1}, \ldots, \epsilon_{r} ; q_{1}^{*}, \ldots, q_{r}^{*}$ are small fixed values.

The local Lipschitz constant of the $F_{\mathbf{G}}(v, \mathbf{q})$-entropy operator (2) and (5) over the set $\mathcal{S}(6)$ is a value $L_{\mathcal{S}}^{F}$ that satisfies the inequality

$$
\begin{gathered}
\left\|\mathbf{u}^{F}\left(v^{1}, \mathbf{q}^{1}\right)-\mathbf{u}^{F}\left(v^{2}, \mathbf{q}^{2}\right)\right\| \leq L_{\mathcal{S}}^{F}\left(\left\|\mathbf{q}^{1}-\mathbf{q}^{2}\right\|+\left\|v^{1}-v^{2}\right\|\right), \\
\left(\mathbf{q}^{1}, v^{1}\right),\left(\mathbf{q}^{2}, v^{2}\right) \in \mathcal{S} .
\end{gathered}
$$

The problem is to find an upper bound $\hat{L}_{\mathcal{S}}^{F}$ for the Lipschitz constant $L_{\mathcal{S}}^{F}$.

The upper bound design method for the Lipschitz constant of the $F_{\mathbf{G}}(v, \mathbf{q})$-entropy operator involves three main ideas as follows. The first idea is to select a suitable operator from the same class for which (1) there exists a close relation to the original operator and (2) it is simpler to obtain an upper bound for the Lipschitz. Such a suitable operator will be called normal and denoted by $F_{\mathbf{G}}^{0}(v, \mathbf{q})$ (Sections 3 and 4$)$. The second idea concerns majorant design: in the beginning, the normal operator $F_{\mathbf{G}}^{0}(\nu, \mathbf{q})$ is majorized by the normal operator $\tilde{B}^{0}(\nu, \mathbf{q})$ (with the Boltzmann entropy) and then an appropriate majorant in form of a linear operator is constructed for it (Section 5). Finally, the third idea deals with the estimation and localization of the eigenvalues of the linear majorant operator (Section 6).

\section{Normal Form of Entropy Operator}

The normal entropy operator $F_{\mathbf{G}}^{0}(v, \mathbf{q})$ is given by

$$
\mathbf{x}^{F}(v, \mathbf{q})=\arg \max _{\mathbf{x}}\left(H_{F}(\mathbf{x}, v) \mid \mathbf{x} \in \mathcal{W} \subset R_{+}^{m}, \mathbf{q} \in \mathcal{Q} \subset R_{+}^{r}, v \in \mathcal{N} \subset R_{+}^{m}\right),
$$

where

$$
H_{F}(\mathbf{x}, v)=-\sum_{i=1}^{m} x_{i} \ln \frac{x_{i}}{v_{i}}+\left(G_{i}-x_{i}\right) \ln \left(G_{i}-x_{i}\right)
$$

denotes the generalized Fermi-Dirac information entropy [7];

$$
\mathcal{W}=\{\mathbf{x}: W \mathbf{x}=\mathbf{q}\}, W \geq 0,
$$

means an admissible set; 
- the matrix $W$ has full rank $r$, normalized columns

$$
W^{\top} \mathbf{e}_{(r)}=\mathbf{e}_{(m)},
$$

$\mathbf{e}_{(r)}, \mathbf{e}_{(m)}$ are unit vectors with $r$ and $m$-dimensions, respectively, and the dominant diagonal of the matrix $W W^{\top}$, i.e.,

$$
\sum_{i=1}^{m} w_{k i}^{2}-\sum_{j \neq k}^{r} \sum_{i=1}^{m} w_{k i} w_{j i} \geq \varrho>0, \quad k=1, \ldots, r
$$

- the definitional domain of the vectors $v, \mathbf{q}$ is

$$
\begin{aligned}
\mathcal{S} & =\mathcal{N} \otimes \mathcal{Q} \\
\mathcal{N} & =\left\{v: 0<\sigma_{i} \leq v_{i} \leq v_{i}^{*}<1 \mid i \in[1, m]\right\} \\
\mathcal{Q} & =\left\{q: 0<\epsilon_{k} \leq q_{k} \leq q_{k}^{*}<\infty \mid k \in[1, r]\right\}
\end{aligned}
$$

with constants $\sigma_{1}, \ldots, \sigma_{m}, v_{1}^{*}, \ldots, v_{m}^{*}$, and $\epsilon_{1}, \ldots, \epsilon_{r} ; q_{1}^{*}, \ldots, q_{r}^{*}$ are small fixed values.

4. Relationship between $F_{\mathbf{G}}(\nu, \mathbf{q})$ and $F_{\mathbf{G}}^{0}(\nu, \mathbf{q})$

Theorem 1. There exists a matrix $P \geq 0$ of dimensions $m \times r$ that satisfies the conditions

$$
\mathbf{e}_{(r)}^{\top} B P=\mathbf{e}_{(r)}
$$

and

$$
\mathbf{u}^{F}(\nu, \mathbf{q})=\mathbf{x}^{F}(\nu, P \mathbf{q})
$$

Proof. Consider system (3):

$$
B \mathbf{u}=\mathbf{q}
$$

Premultiplying this equality by a nondegenerate matrix $P$ of dimensions $m \times r$ yields

$$
P B \mathbf{u}=P \mathbf{q} .
$$

Select the matrix $P$ so that the conditions of Theorem 1 hold. This is a system of $r$ equations with respect to $(m \times r)$ variables-the elements of the matrix $P$. Because the matrix $B$ in (16) is nondegenerate, this system has a set of solutions.

For example, choose the solution that maximizes the entropy

$$
E(P)=-\sum_{i=1}^{m} \sum_{j=1}^{r} p_{i j} \ln p_{i j}
$$

subject to (14).

\section{Majorants of $F_{\mathrm{G}}^{0}(\nu, \mathbf{q})$-Entropy Operator}

Let us use the $B^{0}(v, \mathbf{q})$-entropy operator as a majorant for the $F_{\mathbf{G}}^{0}(v, \mathbf{q})$-entropy operator, defining some domain $\tilde{\mathcal{S}} \subset \mathcal{S}$ (6) where this can be done:

$$
\begin{aligned}
\tilde{\mathcal{S}} & =\tilde{\mathcal{N}} \otimes \tilde{\mathcal{Q}} \\
\tilde{\mathcal{N}} & =\left\{v: 0<\tilde{\sigma}_{i} \leq v_{i} \leq \tilde{v}_{i}^{*}<1, \mid i=\overline{1, m}\right\} \\
\tilde{\mathcal{Q}} & =\left\{q: 0<\tilde{\epsilon}_{k} \leq q_{k} \leq \tilde{q}_{k}^{*}<\infty, \mid k=\overline{1, r}\right\}
\end{aligned}
$$


The $B^{0}(v, \mathbf{q})$-entropy operator has the form

$$
\mathbf{x}^{B}(v, \mathbf{q})=\arg \max _{\mathbf{x}}\left(H_{B}(\mathbf{x}, v) \mid \mathbf{x} \in \mathcal{W} \subset R_{+}^{m}, \mathbf{q} \in \mathcal{Q} \subset R_{+}^{r}, v \in \mathcal{N} \subset R_{+}^{m}\right),
$$

where

$$
H_{B}(\mathbf{x}, v)=-\sum_{i=1}^{m} x_{i} \ln \frac{x_{i}}{v_{i}}
$$

denotes the generalized Boltzmann information entropy.

The characteristics of the admissible set are the same as for the $F_{\mathbf{G}}^{0}(v, \mathbf{q})$-entropy operator (10)-(12). The Lagrange function of the $B^{0}(v, \mathbf{q})$-entropy operator is written as

$$
L(\mathbf{x}, \lambda)=H_{B}(\mathbf{x}, v \mid \mathbf{G})+\sum_{j=1}^{r} \lambda_{j}\left(q_{j}-\sum_{i=1}^{m} w_{j i} x_{i}\right),
$$

where $\lambda_{1}, \ldots, \lambda_{r}$ indicate Lagrange multipliers.

The first-order optimality condition for this function leads to the following system of equations with respect to the dual variables $\mathbf{z}=\exp (-\lambda)$ :

$$
\mathbf{B}(\nu, \mathbf{z}(v, \mathbf{q}))=\mathbf{1} .
$$

Here the vector $\mathbf{B}$ consists of the elements

$$
B_{k}^{0}(v, \mathbf{z}(v, \mathbf{q}))=\frac{1}{q_{k}} \sum_{i=1}^{m} v_{i} w_{k i} \prod_{s=1}^{r} z_{s}^{w_{s i}}, \quad k=\overline{1, r} .
$$

Similar equations hold for the $F_{\mathbf{G}}^{0}(v, \mathbf{q})$-entropy operator. In accordance with (A6),

$$
\mathbf{F}(v, \mathbf{z}(\nu, \mathbf{q}))=\mathbf{1},
$$

where the vector $\mathbf{F}$ consists of the elements

$$
F_{k}^{0}(v, \mathbf{z}(v, \mathbf{q}))=\frac{1}{q_{k}} \sum_{i=1}^{m} \frac{G_{i} t_{k i} \prod_{s=1}^{r} z_{s}^{w_{s i}}}{b_{i}+\prod_{s=1}^{r} z_{s}^{w_{s i}}}, \quad k \overline{1, r} .
$$

Theorem 2. The vector function $\mathbf{B}(v, \mathbf{z}(v, \mathbf{q}))$ (23) and (24) is a majorant in the variable $\mathbf{z}$ for the function $\mathbf{F}(v, \mathbf{z}(v, \mathbf{q}))$ (25) and (26), i.e.,

$$
\mathbf{F}(v, \mathbf{z}(v, \mathbf{q})) \leq \mathbf{B}(v, \mathbf{z}(v, \mathbf{q}))
$$

in the domain $\tilde{\mathcal{S}}$ (19) described by the parameters

$$
\begin{gathered}
\mathbf{z} \geq \mathbf{z}^{0} \in R_{+}^{r}, \quad \mathbf{z}^{0}=\left\{z^{0}, \ldots, z^{0}\right\}, \\
z^{0}=\left(\frac{G^{\min }-\left(1-\sigma^{\min }\right)}{v^{\max }}\right)^{1 / r w^{\max }}, \\
G^{\text {min }}=\min _{i} G_{i}, \sigma^{\min }=\min _{i} \sigma_{i}, v^{\max }=\max _{i} v_{i}^{*}, w^{\max }=\max _{s, i} w_{s i}, \\
\hat{\mathcal{N}}=\left\{v: 0<\sigma^{\min } \leq v_{i} \leq v^{\max }\right\}, \\
\hat{\mathcal{Q}}=\left\{\mathbf{q}: q_{k} \geq \epsilon^{\min }, k=\overline{1, r}\right\}, \\
\epsilon^{\text {min }}=r \frac{G^{\text {min }} w_{\min } z^{0} \sigma^{\min }}{1-\sigma^{\text {min }}+z^{0} \sigma^{\min }}, w_{\text {min }}=\min _{s i} w_{s i} .
\end{gathered}
$$


Proof. Consider inequality (27):

$$
\sum_{i=1}^{m} \frac{G_{i} w_{k i} \prod_{s=1}^{r} z_{s}^{w_{s i}}}{b_{i}+\prod_{s=1}^{r} z_{s}^{w_{s i}}} \leq \sum_{i=1}^{m} v_{i} w_{k i} \prod_{s=1}^{r} z_{s}^{w_{s i}}, \quad k=\overline{1, r} .
$$

Since the terms in the above sums are positive, it suffices that

$$
\frac{G_{i}}{b_{i}+\prod_{s=1}^{r} z_{s}^{w_{s i}}} \leq v_{i}, \quad i=\overline{1, m} .
$$

Consequently,

$$
\prod_{s=1}^{r} z_{s}^{w_{s i}} \geq \prod_{j=1}^{r} v_{i}^{\frac{1}{r}}, \quad v_{i}=\frac{G_{i}-\left(1-v_{i}\right)}{v_{i}} \gg 1, i=\overline{1, m}
$$

This system of inequalities holds if each term in the left-hand side of each inequality is smaller than its counterpart in the right-hand side, i.e.,

$$
z_{s} \geq v_{i}^{1 / r w_{s i}}=h(s, i), \quad s=\overline{1, r} ; i=\overline{1, m} .
$$

Denote

$$
z^{0}=\min _{s, i} h(s, i)=\left(\frac{G^{\min }-\left(1-\sigma_{\min }\right)}{v^{\max }}\right)^{1 / r t_{\max }},
$$

where the variables $G^{\min }, \sigma^{\min }, v^{\max }$, and $t^{\max }$ are defined by Equalities (28).

Now, get back to the system of Equation (25). This system has a nonzero solution $\mathbf{z}^{0}=\left\{z^{0}, \ldots, z^{0}\right\}$ (35) if

$$
q_{k}=\sum_{i=1}^{r} \frac{G_{i} t_{k i} z^{0}}{b_{i}+z^{0}}, \quad k=\overline{1, r} .
$$

Here we have utilized the property (11) of the normal $F_{\mathbf{G}}^{0}(v, \mathbf{q})$-entropy operator. Then, Expression (36) gives

$$
\min _{k}\left(\sum_{i=1}^{r} \frac{G_{i} t_{k i} z^{0}}{b_{i}+z^{0}}\right) \geq r \frac{G^{\min } t_{\min } z^{0} \sigma^{\min }}{1-\sigma^{\min }+z^{0} \sigma^{\min }}=\epsilon_{\min } .
$$

Assume

$$
\epsilon^{\min }<q_{\min }^{*}=\min _{k} q_{k}^{*} .
$$

This condition guarantees the non-emptiness of the set $\mathcal{Q}$ (29). $\tilde{\mathcal{S}}(19)$.

Thus, we have proved that the operator $B^{0}(v, \mathbf{q})$ majorizes the operator $F_{\mathbf{G}}^{0}(v, \mathbf{q})$ in the domain

Theorem 3. For the $B^{0}(v, \mathbf{q})$-entropy operator (20) and (21), there exist two $r$-dimensional positive vectors $\tilde{\mathbf{z}}^{0}$ and $\tilde{\mathbf{z}}^{*}$ such that the nonzero solution $\mathbf{z}(v, \mathbf{q})$ to the system of Equations (23) and (24) belongs to the vector interval

$$
0<\tilde{\mathbf{z}}^{0} \leqq \mathbf{z}(v, \mathbf{q}) \leqq \tilde{\mathbf{z}}^{*}, \quad(v, \mathbf{q}) \in \tilde{\mathcal{S}},
$$

where

- the vector

$$
\tilde{\mathbf{z}}^{0}=\mathbf{z}(\sigma, \epsilon)
$$

is the solution to the system of Equations (23) and (24) that corresponds to the boundary of the set $\tilde{\mathcal{S}}$ (19), i.e., $v=\sigma=\left\{\sigma_{1}, \ldots, \sigma_{m}\right\}$ and $\mathbf{q}=\epsilon=\left\{\epsilon_{1}, \ldots, \epsilon_{r}\right\}$, where $\sigma_{i}$ and $\epsilon_{k}$ are small values; and 
- the vector

$$
\tilde{\mathbf{z}}^{*}=\max _{k=\overline{1, r}} \max _{(v, \mathbf{q}) \in \tilde{\mathcal{S}}} \tilde{z}_{k}(\nu, \mathbf{q}),
$$

where $\tilde{\mathbf{z}}(v, \mathbf{q})$ is the solution to the equation

$$
C(v) \mathbf{z}=\mathbf{q}, \quad \mathbf{z} \geqq 0,
$$

with the matrix

$$
C(v)=\left[c_{k j} \mid(k, j)=\overline{1, r}\right], \quad c_{k j}=\sum_{i=1}^{m} v_{i} w_{k i} w_{j i}>0 .
$$

Proof. Consider Equation (23), reducing it to the form

$$
\mathbf{A}(v, \mathbf{q}, \mathbf{z})=\mathbf{z}, \quad \mathbf{A}(v, \mathbf{q}, \mathbf{z})=\mathbf{z} \otimes \mathbf{B}(v, \mathbf{q}, \mathbf{z}) .
$$

Equation (44) contains a nonnegative strictly monotonically increasing function $\mathbf{A}(v, \mathbf{q}, \mathbf{z})$ in its left-hand side (see [8]). To explore the properties of its solutions, we will employ Theorem 3.1 from [9] for the equations with monotonicoperators. In accordance with this theorem, if there are two vectors $\mathbf{z}^{0} \leqq \mathbf{z}^{*}$ such that

$$
\mathbf{A}\left(v, \mathbf{q}, \mathbf{z}^{0}\right) \geqq \mathbf{z}^{0}, \quad \mathbf{A}\left(\nu, \mathbf{q}, \mathbf{z}^{*}\right) \leqq \mathbf{z}^{*}, \quad(v, \mathbf{q})=\text { fix, }
$$

then the solution to Equation (44) belongs to the vector interval $\left[\mathbf{z}^{0}, \mathbf{z}^{*}\right]$. In our case, $\mathbf{z}^{0}=\mathbf{z}(\sigma, \epsilon)$.

For obtaining an appropriate vector $\mathbf{z}^{*}$, let us construct a majorant for the function $\mathbf{A}(v, \mathbf{q}, \mathbf{z})$ under fixed $v$ and $\mathbf{q}$. Using the inequality

$$
\prod_{i=1}^{r} u_{i}^{\alpha_{i}} \leq \sum_{i=1}^{r} \alpha_{i} u_{i}
$$

where

$$
u_{i} \geq 0, \quad \alpha_{i} \geq 0, \sum_{i=1}^{r} \alpha_{i}=1,
$$

from [10], we get the upper bound

$$
A_{k}(v, \mathbf{q}, \mathbf{z}) \leq z_{k} \frac{1}{q_{k}} \sum_{j=1}^{r} c_{k j} z_{j}, \quad k=\overline{1, r},
$$

where $c_{k j}$ are the elements of the matrix $C$ (43).

By Theorem 3.1 from [9], the nonzero solutions to Equation (44) can be majorized by the nonzero solutionsto Equation (42), i.e.,

$$
\mathbf{z}(\nu, \mathbf{q}) \leqq \tilde{\mathbf{z}}(\nu, \mathbf{q}) \quad \text { under fixed }(\nu, \mathbf{q}) \in \mathcal{S}
$$

\section{Upper Bound $\hat{L}_{\mathcal{S}}^{F}$}

On the strength of Theorems 2 and 3 (also see [8]), we may write the following upper bound for the local Lipschitz constant:

$$
\hat{L}_{\mathcal{S}}^{F}=\frac{x^{\max }}{m}\left(\frac{1}{\sigma^{\min }}+\frac{w_{E}}{M}\right) \text {, }
$$

where

$$
M=x^{\min } \frac{\sigma^{\min }}{v^{\max }} \min _{k}\left(\sum_{i=1}^{m}\left|w_{k i}^{2}-\sum_{j \neq k}^{r} w_{k i} w_{j i}\right|\right),
$$




$$
\begin{gathered}
w_{E}=\sqrt{\sum_{k=1}^{r} \sum_{i=1}^{m} w_{k i^{\prime}}^{2}} \\
x^{\text {min }}=m \frac{G^{\text {min }} v^{\text {min }} z^{0}}{1-v^{\text {max }}+v^{\text {min }} z^{0}}, \\
x^{\text {max }}=m \frac{G^{\text {max }} v^{\text {max }} z^{*}}{1-v^{\text {min }}+v^{\text {max }} z^{*}} .
\end{gathered}
$$

In these formulas, the variables $z^{0}$ and $z^{*}$ are defined by Equalities (40) and (41), respectively.

\section{Conclusions}

The method of upper bound design for the Lipschitz constant of the $F_{\mathbf{G}}(v, \mathbf{q})$-entropy operator is developed. It is based on the normal entropy operator, and the definition of relation between normal and original operators. Then, the linear majorant of the normal operator is defined, and estimation of the Lipschitz constant for the original operator is performed.

The linear majorant method is important for investigation of the properties of entropy operators, for instance, for Boltzmann and Einstein operators [7]. The $F_{\mathbf{G}}(v, \mathbf{q})$-entropy operator is characterized by the parametrical problem for conditional maximization. Also, there exists a wide class of entropy operators that are described by mathematical programming problems. Development of the method of the upper bound design for these operators will represent interesting progress.

Acknowledgments: This work was supported by the Russian Foundation for Basic Research (Project No. 16-07-00743).

Conflicts of Interest: The authors declare no conflict of interest.

\section{Appendix A. Properties of the Normal $F_{G}^{0}(v, q)$-Entropy Operator}

1. Optimality conditions. The Lagrange function of the $F_{v, \mathbf{q}}^{0}$-entropy operator has the form

$$
L(\mathbf{x}, \lambda)=H_{F}(\mathbf{x}, v \mid \mathbf{G})+\sum_{j=1}^{r} \lambda_{j}\left(q_{j}-\sum_{i=1}^{m} w_{j i} x_{i}\right),
$$

where $\lambda_{1}, \ldots, \lambda_{r}$ are Lagrange multipliers.

The first-order optimality conditions of this function yield a system of equations with respect to the direct $\mathbf{x}$ and dual $\lambda$ variables. By the properties of entropy functions, the direct variables possess an analytical relationship to the dual ones, and the optimality conditions are written as the following system of equations with respect to the dual variables (the Lagrange multipliers $\lambda_{1}, \ldots, \lambda_{r}$ ):

$$
\mathbf{x}_{i}^{F}(\nu, \lambda(\nu, \mathbf{q}))=\frac{G_{i} \exp \left(-\sum_{s=1}^{r} \lambda_{s}(\nu, \mathbf{q}) w_{s i}\right)}{b_{i}+\exp \left(-\sum_{s=1}^{r} \lambda_{s}(\nu, \mathbf{q}) w_{s i}\right)}, \quad i \overline{1, m},
$$

where

$$
\begin{gathered}
b_{i}=\frac{1}{v_{i}}, \\
\Omega_{k}^{0}(\nu, \lambda(\nu, \mathbf{q}))=\sum_{i=1}^{m} \frac{G_{i} w_{k i} \exp \left(-\sum_{s=1}^{r} \lambda_{s}(\nu, \mathbf{q}) w_{s i}\right)}{b_{i}+\exp \left(-\sum_{s=1}^{r} \lambda_{s}(v, \mathbf{q}) b_{s i}\right)}=q_{k}, \quad k \overline{1, r} .
\end{gathered}
$$

Sometimes, it is convenient to use similar conditions in terms of the exponential Lagrange multipliers $z_{j}(v, \mathbf{q})=\exp (-\lambda(v, \mathbf{q})) \geq 0, j \in[1, r]$, i.e.,

$$
x_{i}^{F}(v, \mathbf{z}(v, \mathbf{q}))=\frac{G_{i} \prod_{s=1}^{r} z_{s}^{w_{s i}}}{b_{i}+\prod_{s=1}^{r} z_{s}^{w_{s i}}}, \quad i \overline{1, m},
$$




$$
\Theta_{k}^{0}(v, \mathbf{z}(v, \mathbf{q}))=\sum_{i=1}^{m} \frac{G_{i} t_{k i} \prod_{s=1}^{r} z_{s}^{w_{s i}}}{b_{i}+\prod_{s=1}^{r} z_{s}^{w_{s i}}}=q_{k}, \quad k \overline{1, r} .
$$

2. Monotonicity of the function $\Omega_{k}^{0}(\nu, \lambda), \Theta_{k}^{0}(v, \mathbf{z})$.

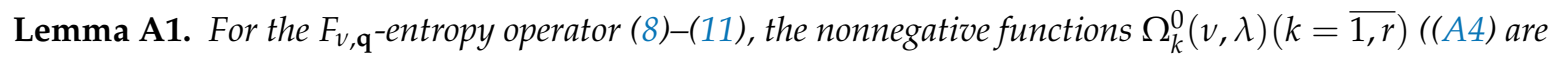
strictly monotonically decreasing in the variables $\left.\lambda \in R^{r}\right)$ and strictly monotonically decreasing in the variables $v \in \mathcal{N}$ while the nonnegative functions $\Theta_{k}^{0}(v, \mathbf{z})(k=\overline{1, r})$ (A6) are strictly monotonically increasing in both variables $v \in \mathcal{N}, \mathbf{z} \in R_{+}^{r}$.

Proof. It suffices to check the signs of corresponding derivatives. Consider Equalities (A4). The derivatives of the function $\Omega_{k}^{0}(v, \lambda)$ with respect to the variables $\lambda$ are given by

$$
\frac{\partial \Omega_{k}^{0}}{\partial \lambda_{j}}=-\sum_{i=1}^{m} \frac{G_{i} b_{i}\left(v_{i}\right) w_{k i} w_{j i} \exp \left(-\sum_{s=1}^{r} \lambda_{s} w_{s i}\right)}{\left[b_{i}+\exp \left(-\sum_{s=1}^{r} \lambda_{s} w_{s i}\right)\right]^{2}} \leq 0, \quad k=\overline{1, r}
$$

Differentiation of the function $\Omega_{k}^{0}(v, \lambda)$ with respect to the variable $v$ yields

$$
\frac{\partial \Omega_{k}^{0}}{\partial v_{p}}=\sum_{i=1}^{m} \frac{G_{i} w_{k i} \exp \left(-\sum_{s=1}^{r} \lambda_{s} w_{s i}\right)}{v_{i}^{2}\left[b_{i}+\exp \left(-\sum_{s=1}^{r} \lambda_{s} w_{s i}\right)\right]^{2}} \geq 0, \quad k=\overline{1, r} .
$$

These expressions vanish if at least one of the variables $\lambda_{p}=+\infty$. This establishes the first part of Lemma A1.

In a similar fashion, for the functions $\Theta_{k}(v, \mathbf{z})(\mathrm{A} 6)$ we obtain

$$
\begin{aligned}
& \frac{\partial \Theta_{k}^{0}}{\partial z_{j}}=\sum_{i=1}^{m} \frac{b_{i} G_{i} w_{k i} t_{j i} z_{j}^{-1} \prod_{s=1}^{r} z_{s}^{w_{s i}}}{\left[b_{i}+\prod_{s=1}^{r} z_{s}^{w_{s i}}\right]^{2}} \geq 0, \quad k=\overline{1, r}, \\
& \frac{\partial \Theta_{k}^{0}}{\partial v_{p}}=\sum_{i=1}^{m} \frac{G_{i} w_{k i} \prod_{s=1}^{r} z_{s}^{w_{s i}}}{v_{i}^{2}\left[b_{i}+\prod_{s=1}^{r} z_{s}^{w_{s i}}\right]^{2}} \geq 0, \quad k=\overline{1, r} .
\end{aligned}
$$

These expressions vanish if at least one of the variables $z_{s}=0$. The proof of Lemma A1 is complete.

Lemma A2. Under the assumptions of Lemma A1, the systems of Equations (A4) and (A6) determine the explicit functions $\lambda(v, \mathbf{q})$ and $\mathbf{z}(v, \mathbf{q})$, respectively.

The proof of this result can be found in [7].

Lemmas $A 1$ and $A 2$ allow us to draw an important conclusion: for two pairs $\left(v^{1}, \mathbf{q}^{1}\right)$ and $\left(v^{2}, \mathbf{q}^{2}\right)$, such that $v^{1} \leq v^{2}$ and $\mathbf{q}^{1} \leq \mathbf{q}^{2}$,

$$
\mathbf{z}\left(v^{1}, \mathbf{q}^{1}\right) \leq \mathbf{z}\left(v^{2}, \mathbf{q}^{2}\right)
$$

\section{References}

1. Herman, G.T. Image Reconstruction from Projections: The Fundamentals of Computerized Tomography; Academic Press: New York, NY, USA, 1980.

2. Lewitt, R.M.; Matej, S. Overview of Methods for Image Reconstruction from Projections in Emission Computed Tomography. Proc. IEEE 2003, 91, 1588-1611. [CrossRef]

3. Popkov, Y.S.; van Wissen, L. Positive Dynamic Systems with Entropy Operator (Application to Labour Market Modeling). Eur. J. Oper. Res. 2005, 164, 811-828. [CrossRef]

4. Popkov, Y.S.; Dubnov, Y.A.; Popkov, A.Y. New Method of Randomized Forecasting Using Entropy-Robust Estimation: Application to the World Population Prediction. Mathematics 2016, 4, 1-16. [CrossRef]

5. Popkov, Y.S. Entropy Operator in Macrosystem Modeling. In Intelligent Systems: From Theory to Practice; SCI 299; Springer: Berlin/Heidelberg, Germany, 2010; pp. 329-359. 
6. Popkov, Y.S. Entropic Image Restoration as a Dynamic System with Entropy Operator. In Image Restoration Recent Advances and Applications; Histace, A., Ed.; INTECH: London, UK, 2012; pp. 45-72.

7. Popkov, Y.S. Macrosystems Theory and Its Applications; Springer: New York, NY, USA, 1995.

8. Popkov, Y.S.; Rublev, M.V. Estimation of a Local Lipschitz Constant of the $B_{q}$-Entropy. Autom. Remote Control 2005, 66, 1069-1080. [CrossRef]

9. Krasnoselskii, M.A.; Vainikko, G.M.; Zabreiko, P.P.; Rutitskii, Y.B.; Stetsenko, V.Y. Approximate Solution of Operator Equations; Nauka: Moscow, Germany, 1969. (In Russian)

10. Beckenbach, E.F.; Bellman, R. Inequalities. Ergebnisse der Mathematik und ihrer Grenzgebiete. 2. Folge; Springer: Berlin/Heidelberg, Germany, 1961; Volume 30.

(C) 2018 by the author. Licensee MDPI, Basel, Switzerland. This article is an open access article distributed under the terms and conditions of the Creative Commons Attribution (CC BY) license (http://creativecommons.org/licenses/by/4.0/). 